\title{
Ground State Solutions for Kirchhoff-type Problems with Critical Nonlinearity
}

\author{
Yiwei Ye
}

Abstract. In this paper, we study the Kirchhoff-type equation with critical exponent

$$
-\left(a+b \int_{\mathbb{R}^{3}}|\nabla u|^{2} d x\right) \Delta u+V(x) u=a(x) f(u)+u^{5} \quad \text { in } \mathbb{R}^{3},
$$

where $a, b>0$ are constants, $V \in C\left(\mathbb{R}^{3}, \mathbb{R}\right), \lim _{|x| \rightarrow \infty} V(x)=V_{\infty}>0$ and $V(x) \leq$ $V_{\infty}+C_{1} e^{-b|x|}$ for some $C_{1}>0$ and $|x|$ large enough. Via variational methods, we prove the existence of ground state solution.

\section{Introduction and main results}

Consider the following Kirchhoff type problem with the critical exponent

$$
-\left(a+b \int_{\mathbb{R}^{3}}|\nabla u|^{2} d x\right) \Delta u+V(x) u=a(x) f(u)+u^{5} \quad \text { in } \mathbb{R}^{3},
$$

where $a, b>0$ are constants.

Problem 1.1) is related to the stationary version of the equation

$$
\begin{cases}u_{t t}-\left(a+b \int_{\Omega}\left|\nabla_{x} u\right|^{2}\right) \Delta_{x} u=f(x, u) & x \in \Omega, t>0, \\ \left.u(\cdot, t)\right|_{\partial \Omega}=0 & t \geq 0\end{cases}
$$

proposed by Kirchhoff [7] in 1883 as an extension of the classical d'Alembert's wave equation for free vibration of elastic strings. Kirchhoff's model takes into account the changes in the length of the string produced by transverse vibrations. In $(1.2), u$ denotes the displacement, $f(x, u)$ the external force and $b$ the initial tension while $a$ is related to the intrinsic properties of the string (such as Young's modulus). We have to point out that Kirchhoff type problems also appear in other fields such as biological systems, where $u$ describes a process which depends on the average of itself (for example, population density). Some early classical studies of Kirchhoff equations were those of Pohozaev [16] and Lions 10,11.

Received August 2, 2017; Accepted March 6, 2019.

Communicated by Jann-Long Chern.

2010 Mathematics Subject Classification. Primary: 45M20; Secondary: 35B33.

Key words and phrases. Kirchhoff-type equation, ground state solutions, variational methods. 
Variational methods have been widely used in the last ten more years in studying the Kirchhoff type problems, see, for instance, [4,6, 14, 15, 20, 21, 26] for results concerning bounded domain, and [1,3,9, 12, 13, 19,22,24] for unbounded domain. Figueiredo [4] studies Kirchhoff type problem on bounded domain with the following version

$$
-M\left(\int_{\Omega}|\nabla u|^{2} d x\right) \Delta u=\lambda f(x, u)+|u|^{2^{*}-2} u \quad \text { in } \Omega
$$

and $u=0$ on $\partial \Omega$, where $\Omega$ is a smooth bounded domain in $\mathbb{R}^{N}$. Applying truncation argument and priori estimates, they prove the existence of positive solutions and their asymptotic behavior depending on $\lambda$. Naimen [14] generalizes Brezis-Nirenberg's result to the Kirchhoff type equation

$$
-\left(a+b \int_{\Omega}|\nabla u|^{2} d x\right) \Delta u=\mu f(x, u)+|u|^{2^{*}-2} u \quad \text { in } \Omega,
$$

where $a, b \geq 0$ and $a+b>0$. Some existence results as well as nonexistence results are obtained. See also 6, 15, 20,26 for related results. For Kirchhoff type equations on the whole space $\mathbb{R}^{3}$, a few results are known. One difficulty is due to the lack of compactness of the embedding from $H^{1}\left(R^{3}\right)$ into $L^{s}\left(R^{3}\right)(2<s<6)$. Alves and Figueiredo [1] study the following periodic Kirchhoff equation with critical growth

$$
M\left(\int_{\mathbb{R}^{N}}|\nabla u|^{2} d x+\int_{\mathbb{R}^{N}} V(x)|u|^{2} d x\right)[-\Delta u+V(x) u]=\lambda f(u)+u^{\tau} \quad \text { in } \mathbb{R}^{N}
$$

where $\tau=5$ for $N=3$ and $\tau \in(1,+\infty)$ for $N=1,2$. Under suitable assumptions on $M$, $V$ and $f$, they construct the existence of positive solutions for $\lambda>0$ large enough. The Kirchhoff type equation with critical nonlinearity of the form

$$
-\left(a+b \int_{\mathbb{R}^{3}}|\nabla u|^{2} d x\right) \Delta u+u=a(x)|u|^{p-2} u+b(x)|u|^{q-2} u+u^{5} \quad \text { in } \mathbb{R}^{3},
$$

has been studied in Zhang 24]. Besides some other conditions, they assume that $a, b \in$ $C\left(\mathbb{R}^{3}, \mathbb{R}\right), \lim _{|x| \rightarrow \infty} a(x)=a_{\infty}, \lim _{|x| \rightarrow \infty} b(x)=0$ and $a(x) \geq a_{\infty}-C e^{-a_{0}|x|}$ for some $a_{0}>0$ and $x \in \mathbb{R}^{3}$ and prove the existence of one ground state solution for $p, q \in(4,6)$ and each $\lambda>0$. It is also proven the existence of two nontrivial solutions for $\lambda>0$ small. Wang et al. 17] discuss the existence, multiplicity and concentration behavior of positive solutions for the following nonlinear Kirchhoff type problem

$$
-\left(\varepsilon^{2} a+\varepsilon b \int_{\mathbb{R}^{3}}|\nabla u|^{2} d x\right) \Delta u+B(x) u=\lambda f(u)+u^{5} \quad \text { in } \mathbb{R}^{3} .
$$

As $\varepsilon \rightarrow 0$, they establish the number of solutions with the topology of the set where $B$ attains its minimum in the case that $f$ is superlinear at zero and subcritical at infinity. 
For more results on the semiclassical limit of $(1.3)$, we refer to $5,19,25]$ and the references therein.

Motivated by the works mentioned above, in this paper, we consider the existence of ground state solution of problem (1.1) in the critical case. We make the following hypotheses:

$\left(\mathrm{V}_{1}\right) \quad V \in C\left(\mathbb{R}^{3}, \mathbb{R}\right), V \geq 0$ on $\mathbb{R}^{3}$ and $\lim _{|x| \rightarrow \infty} V(x)=V_{\infty}>0$.

$\left(\mathrm{V}_{2}\right)$ There exist constants $C_{1}>0, R_{1}>0$ and $b>0$ such that $V(x) \leq V_{\infty}+C_{1} e^{-b|x|}$ for $|x| \geq R_{1}$.

$\left(\mathrm{a}_{1}\right) a \in C\left(\mathbb{R}^{3}, \mathbb{R}\right), a \geq 0$ on $\mathbb{R}^{3}$ and $\lim _{|x| \rightarrow \infty} a(x)=a_{\infty}>0$.

$\left(\mathrm{a}_{2}\right)$ There exist constants $C_{2}>0, R_{2}>0$ and $d>0$ such that $a(x) \geq a_{\infty}+C_{2} e^{-d|x|}$ for $|x| \geq R_{2}$.

$\left(\mathrm{f}_{1}\right) f \in C(\mathbb{R}, \mathbb{R})$ is odd and $\lim _{u \rightarrow 0^{+}} \frac{f(u)}{u}=\lim _{u \rightarrow+\infty} \frac{f(u)}{u^{5}}=0$.

$\left(\mathrm{f}_{2}\right)$ There exist $C_{3}>0$ and $p \in(4,6)$ such that $f(u) \geq C_{3} u^{p-1}$ for all $u \geq 0$.

$\left(\mathrm{f}_{3}\right) f(u) / u^{3}$ is increasing on $(0,+\infty)$.

Theorem 1.1. Assume that $\left(\mathrm{V}_{1}\right)-\left(\mathrm{V}_{2}\right),\left(\mathrm{a}_{1}\right)-\left(\mathrm{a}_{2}\right)$ and $\left(\mathrm{f}_{1}\right)-\left(\mathrm{f}_{3}\right)$ hold with $0<d<b<2$. Then problem (1.1) possesses a ground state solution.

The proof is based on variational method. The main difficulties lie in the appearance of the nonlocal term $\left(\int_{\mathbb{R}^{3}}|\nabla u|^{2} d x\right) \Delta u$, the lack of compactness due to the unboundedness of the domain $\mathbb{R}^{3}$, and the nonlinearity with the critical Sobolev growth. Moreover, since $V(x)$ and $a(x)$ are non-radially symmetric, we cannot restrict the problem in the radially symmetric Sobolev space $H_{r}^{1}\left(\mathbb{R}^{3}\right)$, where the embedding $H_{r}^{1}\left(\mathbb{R}^{3}\right) \hookrightarrow L^{s}\left(\mathbb{R}^{3}\right)(2<s<6)$ is compact. To overcome these difficulties, we have to develop some techniques. In particular, for a sequence $\left(u_{n}\right) \subset E$ with $u_{n} \rightarrow u$ weakly in $E$, we will analyze carefully the difference between $\left(\int_{\mathbb{R}^{3}}\left|\nabla u_{n}\right|^{2} d x\right)^{2}$ and $\left(\int_{\mathbb{R}^{3}}|\nabla u|^{2} d x\right)^{2}$ to prove the $(P S)_{c}$ condition holds for a suitable range of $c$.

The paper is organized as follows. In Section 2, we give some preliminaries and prove the local Palais-Smale condition of the functional corresponding to (1.1). In Section 3 . we will analyze the term $\int_{\mathbb{R}^{3}} V(x) u^{2} d x$ delicately and its competing with the nonlinearity $\int_{\mathbb{R}^{3}} a(x) F(u) d x$, where $F(u)=\int_{0}^{u} f(s) d s$, and prove Theorem 1.1 .

\section{Notations}

- For any $1 \leq s \leq+\infty$, we denote by $\|\cdot\|_{s}$ the usual norm of the Lebesgue space $L^{s}\left(\mathbb{R}^{3}\right)$. 
- $H^{1}\left(\mathbb{R}^{3}\right)$ is the usual Hilbert space endowed with the norm $\|u\|_{H^{1}}^{2}=\int_{\mathbb{R}^{3}}\left(|\nabla u|^{2}+\right.$ $\left.u^{2}\right) d x$.

- $\mathcal{D}^{1,2}\left(\mathbb{R}^{3}\right)$ is the completion of $C_{0}^{\infty}\left(\mathbb{R}^{3}\right)$ with respect to the norm $\|u\|_{\mathcal{D}^{1,2}}^{2}:=\int_{\mathbb{R}^{3}}|\nabla u|^{2}$ $d x$.

- $S$ denotes the best Sobolev constant

$$
S:=\inf _{u \in \mathcal{D}^{1,2} \backslash\{0\}} \frac{\|\nabla u\|_{2}^{2}}{\|u\|_{2^{*}}^{2}} .
$$

- $C$ and $C_{i}$ denote various positive constants, which may vary from line to line.

\section{Preliminary results}

In this section, we assume $\left(\mathrm{V}_{1}\right),\left(\mathrm{a}_{1}\right)$ and $\left(\mathrm{f}_{1}\right)-\left(\mathrm{f}_{3}\right)$ hold. Let

$$
E=\left\{u \in H^{1}\left(\mathbb{R}^{3}\right): \int_{\mathbb{R}^{3}} V(x) u^{2} d x<+\infty\right\} .
$$

It is easy to recognize that $E$ is the Hilbert space with the inner product and norm

$$
(u, v)=\int_{\mathbb{R}^{3}}(a \nabla u \cdot \nabla v+V(x) u v) d x, \quad\|u\|=(u, u)^{1 / 2} .
$$

From $\left(\mathrm{V}_{1}\right)$ and $\left(\mathrm{a}_{1}\right)$, there exists a constant $M>0$ such that

$$
|V(x)| \leq M, \quad|a(x)| \leq M, \quad \forall x \in \mathbb{R}^{3},
$$

and there exists $R_{0}>0$ such that $V(x) \geq V_{\infty} / 2$ for $|x| \geq R_{0}$. Hence we have

$$
\begin{aligned}
& \int_{\mathbb{R}^{3}}\left(|\nabla u|^{2}+u^{2}\right) d x \\
\leq & \int_{|x| \leq R_{0}}|\nabla u|^{2} d x+\int_{|x| \leq R_{0}} u^{2} d x+\int_{|x| \geq R_{0}}\left(|\nabla u|^{2}+\frac{2}{V_{\infty}} V(x) u^{2}\right) d x \\
\leq & \int_{|x| \leq R_{0}}|\nabla u|^{2} d x+\left(\int_{|x| \leq R_{0}} u^{6} d x\right)^{1 / 3}\left|\int_{|x| \leq R_{0}} 1 d x\right|^{2 / 3} \\
& +\left(\frac{1}{a}+\frac{2}{V_{\infty}}\right) \int_{|x| \geq R_{0}}\left(a|\nabla u|^{2}+V(x) u^{2}\right) d x \\
\leq & \max \left\{\frac{1}{a}+(a S)^{-1}\left|\int_{|x| \leq R_{0}} 1 d x\right|^{2 / 3}, \frac{1}{a}+\frac{2}{V_{\infty}}\right\}\|u\|^{2} \\
\leq & \left(\frac{1}{a}+(a S)^{-1}\left|\int_{|x| \leq R_{0}} 1 d x\right|^{2 / 3}+\frac{2}{V_{\infty}}\right)\|u\|^{2},
\end{aligned}
$$


which implies that the embedding $E \hookrightarrow H^{1}\left(\mathbb{R}^{3}\right)$ is continuous. Consider the functional $I: E \rightarrow \mathbb{R}$ defined by

$$
I(u)=\frac{1}{2}\|u\|^{2}+\frac{b}{4}\left(\int_{\mathbb{R}^{3}}|\nabla u|^{2} d x\right)^{2}-\int_{\mathbb{R}^{3}}\left(a(x) F(u)+\frac{1}{6} u^{6}\right) d x .
$$

It is easy to check that $I \in C^{1}(E, \mathbb{R})$ and $u \in E$ is a solution of problem (1.1) if and only if $u$ is a critical point of $I$. Define the functional

$$
I^{\infty}(u)=\frac{1}{2}\|u\|_{V_{\infty}}^{2}+\frac{b}{4}\left(\int_{\mathbb{R}^{3}}|\nabla u|^{2} d x\right)^{2}-\int_{\mathbb{R}^{3}}\left(a_{\infty} F(u)+\frac{1}{6}|u|^{6}\right) d x, \quad \forall u \in E,
$$

where $\|u\|_{V_{\infty}}^{2}:=\int_{\mathbb{R}^{3}}\left(a|\nabla u|^{2}+V_{\infty}|u|^{2}\right) d x$. Take

$$
m_{\infty}=\inf \left\{I^{\infty}(u): u \in M_{\infty}\right\}
$$

with $M_{\infty}=\left\{u \in E \backslash\{0\}: I^{\infty \prime}(u)=0\right\}$. According to [12, Theorem 1.3], $m_{\infty}$ is obtained by a positive function $u_{\infty}$ and $I^{\infty \prime}\left(u_{\infty}\right)=0$. Similar arguments as in 23, Lemma 2.5], we can deduce that for any $\delta>0$, there exists $C_{\delta}>0$ such that

$$
u_{\infty}(x) \leq C_{\delta} e^{-(1-\delta) \sqrt{V_{\infty}}|x|}, \quad \forall x \in \mathbb{R}^{3} .
$$

By $\left(\mathrm{f}_{1}\right)$, we deduce that for any $\varepsilon>0$, there exists $C_{\varepsilon}>0$ such that

$$
\max \{|F(u)|,|f(u) u|\} \leq \varepsilon|u|^{2}+C_{\varepsilon}|u|^{6}, \quad \forall u \in \mathbb{R}
$$

and

$$
\max \{|F(u)|,|f(u) u|\} \leq \varepsilon\left(|u|^{2}+|u|^{6}\right)+C_{\varepsilon}|u|^{s}, \quad \forall u \in \mathbb{R},
$$

where $s \in(2,6)$. From $\left(\mathrm{f}_{3}\right)$, we derive that

$$
\frac{1}{4} f(u) u-F(u) \geq 0, \quad \forall u \in \mathbb{R}
$$

and $\frac{1}{4} f(u) u-F(u)$ is increasing for $u \geq 0$.

For $\varepsilon>0$, let

$$
v_{\varepsilon}(x)=\frac{\psi(x) \varepsilon^{1 / 4}}{\left(\varepsilon+|x|^{2}\right)^{1 / 2}},
$$

where $\psi \in C_{0}^{\infty}\left(\mathbb{R}^{3},[0,1]\right)$ such that $\psi(x)=1$ for $|x| \leq r_{0}$ and $\psi(x)=0$ for $|x| \geq 2 r_{0}$. It is well known that $S$ is attained by the function $\varepsilon^{1 / 4} /\left(\varepsilon+|x|^{2}\right)^{1 / 2}$. Direct calculation shows that (see [18])

$$
\begin{aligned}
\int_{\mathbb{R}^{3}}\left|\nabla v_{\varepsilon}\right|^{2} d x & =\int_{\mathbb{R}^{3}} \frac{|x|^{2}}{\left(1+|x|^{2}\right)^{3}} d x+O\left(\varepsilon^{1 / 2}\right):=K_{1}+O\left(\varepsilon^{1 / 2}\right), \\
\int_{\mathbb{R}^{3}}\left|v_{\varepsilon}\right|^{6} d x & =\int_{\mathbb{R}^{3}} \frac{1}{\left(1+|x|^{2}\right)^{3}} d x:=K_{2}+O\left(\varepsilon^{3 / 2}\right)
\end{aligned}
$$


and

$$
\int_{\mathbb{R}^{3}}\left|v_{\varepsilon}\right|^{s} d x= \begin{cases}O\left(\varepsilon^{(6-s) / 4}\right) & s \in(3,6), \\ O\left(\varepsilon^{3 / 4}|\ln \varepsilon|\right) & s=3 \\ O\left(\varepsilon^{s / 4}\right) & s \in[2,3),\end{cases}
$$

where $K_{1}, K_{2}$ are positive constants and $S=K_{1} / K_{2}^{1 / 3}$.

Lemma 2.1. $m_{\infty}<c^{*}:=\frac{a}{3}\left\{\frac{b S^{3}+\sqrt{\left(b S^{3}\right)^{2}+4 a S^{3}}}{2}\right\}+\frac{b}{12}\left\{\frac{b S^{3}+\sqrt{\left(b S^{3}\right)^{2}+4 a S^{3}}}{2}\right\}^{2}$.

Proof. Let $c_{\infty}=\inf _{\gamma \in \Gamma_{\infty}} \max _{t \in[0,1]} I^{\infty}(\gamma(t))$, where $\Gamma_{\infty}=\{\gamma \in C([0,1], E): \gamma(0)=$ $\left.0, I^{\infty}(\gamma(1))<0\right\}$. Similar to 18 , Theorems 4.1 and 4.2$]$, we have

$$
m_{\infty}=c_{\infty}=\inf _{u \in E \backslash\{0\}} \max _{t \geq 0} I^{\infty}(t u)>0 .
$$

Hence $m_{\infty} \leq \sup _{t \geq 0} I^{\infty}\left(t v_{\varepsilon}\right)$. To obtain $m_{\infty}<c^{*}$, it suffices to prove that $\sup _{t \geq 0} I^{\infty}\left(t v_{\varepsilon}\right)$ $<c^{*}$.

It follows from (2.7) and (2.8) that there exists $\varepsilon_{1}>0$ such that for $\varepsilon \in\left(0, \varepsilon_{1}\right)$,

$$
\int_{\mathbb{R}^{3}}\left|\nabla v_{\varepsilon}\right|^{2} d x<\frac{3 K_{1}}{2}, \quad \int_{\mathbb{R}^{3}}\left|v_{\varepsilon}\right|^{2} d x \leq \frac{1}{2}, \quad \int_{\mathbb{R}^{3}}\left|v_{\varepsilon}\right|^{6} d x \geq \frac{K_{2}}{2} .
$$

Thus, for $\varepsilon \in\left(0, \varepsilon_{1}\right)$,

$$
\begin{aligned}
I^{\infty}\left(t v_{\varepsilon}\right) & \leq \frac{t^{2}}{2}\left\|v_{\varepsilon}\right\|_{V_{\infty}}^{2}+\frac{b t^{4}}{4}\left(\int_{\mathbb{R}^{3}}\left|\nabla v_{\varepsilon}\right|^{2} d x\right)^{2}-\frac{t^{6}}{6} \int_{\mathbb{R}^{3}}\left|v_{\varepsilon}\right|^{6} d x \\
& \leq \frac{\left(3 a K_{1}+V_{\infty}\right) t^{2}}{4}+\frac{9 b K_{1}^{2} t^{4}}{16}-\frac{K_{2} t^{6}}{12},
\end{aligned}
$$

which implies that there exist $t_{1}>0$ small and $t_{2}>0$ large (independent of $\varepsilon$ ) such that

$$
\sup _{t \in\left[0, t_{1}\right] \cup\left[t_{2},+\infty\right]} I^{\infty}\left(t v_{\varepsilon}\right)<c^{*} .
$$

Take

$$
A_{\varepsilon}=\frac{\int_{\mathbb{R}^{3}} a\left|\nabla v_{\varepsilon}\right|^{2} d x}{\left(\int_{\mathbb{R}^{3}}\left|v_{\varepsilon}\right|^{6} d x\right)^{1 / 3}} \quad \text { and } \quad B_{\varepsilon}=\frac{b\left(\int_{\mathbb{R}^{3}}\left|\nabla v_{\varepsilon}\right|^{2} d x\right)^{2}}{\left(\int_{\mathbb{R}^{3}}\left|v_{\varepsilon}\right|^{6} d x\right)^{2 / 3}} .
$$

From (2.7), one has $A_{\varepsilon}=a S+O\left(\varepsilon^{1 / 2}\right)$ and $B_{\varepsilon}=b S^{2}+O\left(\varepsilon^{1 / 2}\right)$. Then a direct calculation shows that

$$
\begin{aligned}
& \sup _{t \geq 0}\left[\frac{t^{2}}{2} \int_{\mathbb{R}^{3}} a\left|\nabla v_{\varepsilon}\right|^{2} d x+\frac{b t^{4}}{4}\left(\int_{\mathbb{R}^{3}}\left|\nabla v_{\varepsilon}\right|^{2} d x\right)^{2}-\frac{t^{6}}{6} \int_{\mathbb{R}^{3}}\left|v_{\varepsilon}\right|^{6} d x\right] \\
= & \frac{1}{4} A_{\varepsilon}\left(B_{\varepsilon}+\sqrt{B_{\varepsilon}^{2}+4 A_{\varepsilon}}\right)+\frac{1}{16} B_{\varepsilon}\left(B_{\varepsilon}+\sqrt{B_{\varepsilon}^{2}+4 A_{\varepsilon}}\right)^{2}-\frac{1}{48}\left(B_{\varepsilon}+\sqrt{B_{\varepsilon}^{2}+4 A_{\varepsilon}}\right)^{3} \\
= & \frac{1}{6} A_{\varepsilon}\left(B_{\varepsilon}+\sqrt{B_{\varepsilon}^{2}+4 A_{\varepsilon}}\right)+\frac{1}{48} B_{\varepsilon}\left(B_{\varepsilon}+\sqrt{B_{\varepsilon}^{2}+4 A_{\varepsilon}}\right)^{2} \\
= & c^{*}+O\left(\varepsilon^{1 / 2}\right) .
\end{aligned}
$$


Hence, combining 2.10, 2.8 and $\left(\mathrm{f}_{2}\right)$, we obtain

$$
\begin{aligned}
\sup _{t \in\left[t_{1}, t_{2}\right]} I^{\infty}\left(t v_{\varepsilon}\right) \leq & \sup _{t \geq 0}\left[\frac{t^{2}}{2} \int_{\mathbb{R}^{3}} a\left|\nabla v_{\varepsilon}\right|^{2} d x+\frac{b t^{4}}{4}\left(\int_{\mathbb{R}^{3}}\left|\nabla v_{\varepsilon}\right|^{2} d x\right)^{2}-\frac{t^{6}}{6} \int_{\mathbb{R}^{3}}\left|v_{\varepsilon}\right|^{6} d x\right] \\
& +\frac{V_{\infty} t_{2}^{2}}{2} \int_{\mathbb{R}^{3}}\left|v_{\varepsilon}\right|^{2} d x-\frac{C_{3} a_{\infty} t_{1}^{p}}{p} \int_{\mathbb{R}^{3}}\left|v_{\varepsilon}\right|^{p} d x \\
= & c^{*}+O\left(\varepsilon^{1 / 2}\right)-O\left(\varepsilon^{(6-p) / 4}\right) .
\end{aligned}
$$

Observing $(6-p) / 4<1 / 2$, choosing $\varepsilon \in\left(0, \varepsilon_{1}\right)$ sufficiently small, we get $\sup _{t \in\left[t_{1}, t_{2}\right]} I^{\infty}\left(t v_{\varepsilon}\right)$ $<c^{*}$, which, jointly with 2.9$)$, shows that $\sup _{t \geq 0} I^{\infty}\left(t v_{\varepsilon}\right)<c^{*}$. This completes the proof.

Recall that, for $c \in \mathbb{R}$, a $(P S)_{c}$-sequence for the functional $I$ is referred to a sequence $\left(u_{n}\right) \subset E$ such that $I\left(u_{n}\right) \rightarrow c$ and $I^{\prime}\left(u_{n}\right) \rightarrow 0$ in $E^{*}$ (the dual space of $E$ ). $I$ is said to satisfy $(P S)_{c}$ condition if any $(P S)_{c}$ sequence has a convergent subsequence.

Lemma 2.2. Suppose that $\left(u_{n}\right)$ be a $(P S)_{c}$-sequence of $I$ with $c \in\left(0, m_{\infty}\right)$. Then $\left(u_{n}\right)$ possesses a strongly convergent subsequence.

Proof. It follows from $\left(\mathrm{a}_{1}\right)$ and $(2.6)$ that

$$
c+o(1)+o(1)\left\|u_{n}\right\|=I\left(u_{n}\right)-\frac{1}{4}\left\langle I^{\prime}\left(u_{n}\right), u_{n}\right\rangle \geq \frac{1}{4}\left\|u_{n}\right\|^{2},
$$

which implies that $\left(u_{n}\right)_{n \in \mathbb{N}}$ is bounded. Going if necessary to a subsequence, still denoted by $\left(u_{n}\right)$, we may assume that there is $u \in E$ such that for each bounded domain $\Omega \subset \mathbb{R}^{3}$,

$$
\begin{aligned}
u_{n} & \rightarrow u \text { in } E, \quad \nabla u_{n} \rightarrow \nabla u \text { in } L^{2}\left(\mathbb{R}^{3}\right), \\
u_{n} & \rightarrow u \text { in } L^{s}(\Omega) \quad(2<s<6), \\
u_{n}(x) & \rightarrow u(x) \text { a.e. } x \in \mathbb{R}^{3} .
\end{aligned}
$$

Set $A=\lim _{n \rightarrow \infty} \int_{\mathbb{R}^{3}}\left|\nabla u_{n}\right|^{2} d x$. We define the functionals $J, H, \Phi, \Psi$ on $E$ by

$$
\begin{aligned}
& J(u)=\frac{1}{2}\|u\|^{2}+\frac{b A}{2} \int_{\mathbb{R}^{3}}|\nabla u|^{2} d x-\int_{\mathbb{R}^{3}}\left(a(x) F(u)+\frac{1}{6} u^{6}\right) d x, \\
& H(u)=\frac{1}{2}\|u\|^{2}+\frac{b A}{4} \int_{\mathbb{R}^{3}}|\nabla u|^{2} d x-\int_{\mathbb{R}^{3}}\left(a(x) F(u)+\frac{1}{6} u^{6}\right) d x, \\
& \Phi(u)=\frac{1}{2}\|u\|_{V_{\infty}}^{2}+\frac{b A}{2} \int_{\mathbb{R}^{3}}|\nabla u|^{2} d x-\int_{\mathbb{R}^{3}}\left(a_{\infty} F(u)+\frac{1}{6} u^{6}\right) d x, \\
& \Psi(u)=\frac{1}{2}\|u\|_{V_{\infty}}^{2}+\frac{b A}{4} \int_{\mathbb{R}^{3}}|\nabla u|^{2} d x-\int_{\mathbb{R}^{3}}\left(a_{\infty} F(u)+\frac{1}{6} u^{6}\right) d x .
\end{aligned}
$$


We claim that $J^{\prime}(u)=0$, i.e., $\left\langle J^{\prime}(u), \varphi\right\rangle=0$ for any $\varphi \in C_{0}^{\infty}\left(\mathbb{R}^{3}\right)$. Assume that $1 \leq$ $p, q, r, s<+\infty, \Omega$ is a bounded domain and $h \in C(\Omega \times \mathbb{R})$ satisfying $|h(x, u)| \leq C\left(|u|^{p / r}+\right.$ $\left.|u|^{q / s}\right)$, then, according to $[18$, Theorem A.4], the operator

$$
A: L^{p}(\Omega) \cap L^{q}(\Omega) \rightarrow L^{r}(\Omega)+L^{s}(\Omega): u \rightarrow h(x, u)
$$

is continuous, where $L^{p}(\Omega) \cap L^{q}(\Omega)$ is the space endowed with the norm $|u|_{p \wedge q}=\|u\|_{L^{p}(\Omega)}+$ $\|u\|_{L^{q}(\Omega)}$ and $L^{r}(\Omega)+L^{s}(\Omega)$ endowed with the norm

$$
|u|_{r \vee s}=\inf \left\{\|v\|_{L^{r}(\Omega)}+\|w\|_{L^{s}(\Omega)}: u=v+w, v \in L^{r}(\Omega), w \in L^{s}(\Omega)\right\} .
$$

Now set $p=r=2, q \in(5,6), s=q / 5$ and $h(x, u)=a(x) f(u)+u^{5}$. By $\left(\mathrm{a}_{1}\right)$ and $\left(\mathrm{f}_{1}\right)$, we have

$$
|h(x, u)| \leq C\left(|u|^{2 / 2}+|u|^{q / s}\right), \quad \forall(x, u) \in \mathbb{R}^{3} \times \mathbb{R} .
$$

Since $\varphi \in C_{0}^{\infty}\left(\mathbb{R}^{3}\right)$ has a compact support $\Omega_{0}$. $u_{n} \rightarrow u$ in $E$ implies that $u_{n} \rightarrow u$ in $L^{2}\left(\Omega_{0}\right) \cap L^{q}\left(\Omega_{0}\right)$. So, by virtue of $[18$, Theorem A.4],

$$
h\left(x, u_{n}\right) \rightarrow h(x, u) \quad \text { in } L^{2}\left(\Omega_{0}\right)+L^{s}\left(\Omega_{0}\right) .
$$

Hence

$$
\begin{aligned}
\int_{\mathbb{R}^{3}}\left|\left(h\left(x, u_{n}\right)-h(x, u)\right) \varphi\right| d x & =\int_{\Omega_{0}}\left|\left(h\left(x, u_{n}\right)-h(x, u)\right) \varphi\right| d x \\
& \leq\left|h\left(x, u_{n}\right)-h(x, u)\right|_{2 \vee s}|\varphi|_{2 \wedge s^{\prime}} \stackrel{n}{\longrightarrow} 0
\end{aligned}
$$

where $1 / s+1 / s^{\prime}=1$. Combining this and 2.11), we get that $o(1)=\left\langle I^{\prime}\left(u_{n}\right), \varphi\right\rangle=$ $\left\langle J^{\prime}(u), \varphi\right\rangle+o(1)$ for any $\varphi \in C_{0}^{\infty}\left(\mathbb{R}^{3}\right)$. Thus $J^{\prime}(u)=0$.

Let $v_{n}:=u_{n}-u$. It follows from [27, Lemma 2.2] and Brezis-Lieb lemma that

$$
\int_{\mathbb{R}^{3}} a(x)\left(F\left(u_{n}\right)-F(u)-F\left(v_{n}\right)\right) d x \rightarrow 0
$$

and

$$
\int_{\mathbb{R}^{3}}\left(u_{n}^{6}-u^{6}-v_{n}^{6}\right) d x \rightarrow 0
$$

as $n \rightarrow \infty$. Since $v_{n} \rightarrow 0$ in $E$, by $\left(\mathrm{V}_{1}\right),\left(\mathrm{a}_{1}\right)$ and $\left(\mathrm{f}_{1}\right)$, we obtain that

$$
\int_{\mathbb{R}^{3}}\left(V(x)-V_{\infty}\right) v_{n}^{2} d x \rightarrow 0, \quad \int_{\mathbb{R}^{3}}\left(a(x)-a_{\infty}\right) F\left(v_{n}\right) d x \rightarrow 0
$$

and

$$
\int_{\mathbb{R}^{3}}\left(V(x)-V_{\infty}\right) v_{n} \xi d x \rightarrow 0, \quad \int_{\mathbb{R}^{3}}\left(a(x)-a_{\infty}\right) f\left(v_{n}\right) \xi d x \rightarrow 0, \quad \forall \xi \in E
$$


as $n \rightarrow \infty$. Hence, using 2.14, 2.13) and 2.12, we deduce that

$$
\begin{aligned}
c+o(1)= & I\left(u_{n}\right) \\
= & \frac{1}{2}\left(\|u\|^{2}+\left\|v_{n}\right\|^{2}\right)+\frac{b A}{4} \int_{\mathbb{R}^{3}}\left(|\nabla u|^{2}+\left|\nabla v_{n}\right|^{2}\right) d x \\
& -\int_{\mathbb{R}^{3}}\left(a(x) F(u)+a(x) F\left(v_{n}\right)\right) d x-\int_{\mathbb{R}^{3}} \frac{1}{6}\left(u^{6}+v_{n}^{6}\right) d x+o(1) \\
= & \frac{1}{2}\left(\|u\|^{2}+\left\|v_{n}\right\|_{V_{\infty}}^{2}\right)+\frac{b A}{4} \int_{\mathbb{R}^{3}}\left(|\nabla u|^{2}+\left|\nabla v_{n}\right|^{2}\right) d x \\
& -\int_{\mathbb{R}^{3}}\left(a(x) F(u)+a_{\infty} F\left(v_{n}\right)\right) d x-\int_{\mathbb{R}^{3}} \frac{1}{6}\left(u^{6}+v_{n}^{6}\right) d x+o(1) \\
= & H(u)+\Psi\left(v_{n}\right)+o(1) .
\end{aligned}
$$

Moreover, noticing $J^{\prime}(u)=0$, by $\left(\mathrm{a}_{1}\right)$ and $(2.6)$ we have

$$
\begin{aligned}
H(u) & =H(u)-\frac{1}{4}\left\langle J^{\prime}(u), u\right\rangle \\
& =\frac{1}{4}\|u\|^{2}+\int_{\mathbb{R}^{3}}\left[a(x)\left(\frac{1}{4} f(u) u-F(u)\right)+\frac{1}{12} u^{6}\right] d x \geq 0 .
\end{aligned}
$$

From [18, Lemma 8.9], we get

$$
\left|\int_{\mathbb{R}^{3}}\left(u_{n}^{5}-u^{5}-v_{n}^{5}\right) \xi d x\right|=o(1)\|\xi\|, \quad \forall \xi \in E .
$$

Similar to [18, Lemma 8.1], one has

$$
\left|\int_{\mathbb{R}^{3}} a(x)\left(f\left(u_{n}\right)-f(u)-f\left(v_{n}\right)\right) \xi d x\right|=o(1)\|\xi\|, \quad \forall \xi \in E .
$$

Combining (2.19), 2.18 and (2.15) and using the fact $J^{\prime}(u)=0$, we obtain

$$
\begin{aligned}
o(1) & =\left\langle I^{\prime}\left(u_{n}\right), \xi\right\rangle-\left\langle J^{\prime}(u), \xi\right\rangle \\
& =\left(v_{n}, \xi\right)+b A \int_{\mathbb{R}^{3}} \nabla v_{n} \nabla \xi d x-\int_{\mathbb{R}^{3}}\left(a(x) f\left(v_{n}\right) \xi+v_{n}^{5} \xi\right) d x+o(1) \\
& =\int_{\mathbb{R}^{3}}\left(a \nabla v_{n} \nabla \xi+V_{\infty} v_{n} \xi\right) d x+b A \int_{\mathbb{R}^{3}} \nabla v_{n} \nabla \xi d x-\int_{\mathbb{R}^{3}}\left(a_{\infty} f\left(v_{n}\right) \xi+v_{n}^{5} \xi\right) d x+o(1) \\
& =\left\langle\Phi^{\prime}\left(v_{n}\right), \xi\right\rangle+o(1), \quad \forall \xi \in E,
\end{aligned}
$$

which implies that

$$
\Phi^{\prime}\left(v_{n}\right)=o(1)
$$

Next we prove that $v_{n} \rightarrow 0$ in $E$. Let $\rho_{n}(x)=\left|v_{n}(x)\right|^{2}$. Then by [8, Lemma 2.1], for some subsequence of $\left\{\rho_{n}(x)\right\}$, either "vanishing" or "nonvanishing" holds. If "nonvanishing" occurs, there exist $\sigma>0, r>0$ and $\left(y_{n}\right) \subset \mathbb{R}^{3}$ such that

$$
\liminf _{n \rightarrow \infty} \int_{B_{r}\left(y_{n}\right)}\left|v_{n}(x)\right|^{2} d x \geq \sigma>0 .
$$


Let $\widetilde{v}_{n}(x)=v_{n}\left(x+y_{n}\right)$. We claim that

$$
\Phi^{\prime}\left(\widetilde{v}_{n}\right)=o(1)
$$

Indeed, for all $\xi \in E$, set $\xi_{n}(x)=\xi\left(x-y_{n}\right)$. It is easy to see that $\left\|\xi_{n}\right\|_{H^{1}}=\|\xi\|_{H^{1}}$, therefore, by (2.20) and (2.1),

$$
\begin{aligned}
\left|\left\langle\Phi^{\prime}\left(\widetilde{v}_{n}\right), \xi\right\rangle\right| & =\left|\int_{\mathbb{R}^{3}}\left(a \nabla \widetilde{v}_{n} \cdot \nabla \xi+V_{\infty} \widetilde{v}_{n} \xi+b A \nabla \widetilde{v}_{n} \cdot \nabla \xi-a_{\infty} f\left(\widetilde{v}_{n}\right) \xi+\widetilde{v}_{n}^{5} \xi\right) d x\right| \\
& =\left|\int_{\mathbb{R}^{3}}\left(a \nabla v_{n} \cdot \nabla \xi_{n}+V_{\infty} v_{n} \xi_{n}+b A \nabla v_{n} \cdot \nabla \xi_{n}-a_{\infty} f\left(v_{n}\right) \xi_{n}+v_{n}^{5} \xi_{n}\right) d x\right| \\
& =\left|\left\langle\Phi^{\prime}\left(v_{n}\right), \xi_{n}\right\rangle\right| \leq\left\|\Phi^{\prime}\left(v_{n}\right)\right\|\left\|\xi_{n}\right\| \leq C\left\|\Phi^{\prime}\left(v_{n}\right)\right\|\left\|\xi_{n}\right\|_{H^{1}} \\
& \leq C\left\|\Phi^{\prime}\left(v_{n}\right)\right\|\|\xi\|_{H^{1}} \stackrel{n}{\longrightarrow} 0,
\end{aligned}
$$

and 2.22 is proved. Since $\left(v_{n}\right)$ is bounded in $E,\left(\widetilde{v}_{n}\right)$ is also bounded in $E$. Then we may assume that $\widetilde{v}_{n} \rightarrow \widetilde{v}$ in $E$ and $\widetilde{v} \neq 0$ by (2.21). Observing $\Phi^{\prime}$ is weakly continuous, it follows that $\Phi^{\prime}(\widetilde{v})=0$, so

$$
\|\widetilde{v}\|_{V_{\infty}}^{2}+b A \int_{\mathbb{R}^{3}}|\nabla \widetilde{v}|^{2} d x=\int_{\mathbb{R}^{3}}\left(a_{\infty} f(\widetilde{v}) \widetilde{v}+\widetilde{v}^{6}\right) d x
$$

For $\widetilde{v} \in E \backslash\{0\}$, there exists a unique $t>0$ such that $t \widetilde{v} \in M_{\infty}$, i.e.,

$$
t^{2}\|\widetilde{v}\|_{V_{\infty}}^{2}+b t^{4}\left(\int_{\mathbb{R}^{3}}|\nabla \widetilde{v}|^{2} d x\right)^{2}=\int_{\mathbb{R}^{3}}\left(a_{\infty} f(t \widetilde{v}) t \widetilde{v}+t^{6} \widetilde{v}^{6}\right) d x .
$$

We claim that $t \leq 1$. If $t>1$, by $(2.24),(2.23)$ and $\left(\mathrm{f}_{3}\right)$ and using the fact $A \geq \int_{\mathbb{R}^{3}}|\nabla \widetilde{v}|^{2} d x$, we deduce that

$$
\begin{aligned}
& t^{4} \int_{\mathbb{R}^{3}}\left(a_{\infty} f(\widetilde{v}) \widetilde{v}+\widetilde{v}^{6}\right) d x \\
\leq & \int_{\mathbb{R}^{3}}\left(t^{4} a_{\infty} f(\widetilde{v}) \widetilde{v}+t^{6} \widetilde{v}^{6}\right) d x \leq \int_{\mathbb{R}^{3}}\left(a_{\infty} \frac{f(t \widetilde{v})}{(t \widetilde{v})^{3}} t^{4} \widetilde{v}^{4}+t^{6} \widetilde{v}^{6}\right) d x \\
= & t^{2}\|\widetilde{v}\|_{V_{\infty}}^{2}+b t^{4}\left(\int_{\mathbb{R}^{3}}|\nabla \widetilde{v}|^{2} d x\right)^{2}<t^{4}\left(\|\widetilde{v}\|_{V_{\infty}}+b\left(\int_{\mathbb{R}^{3}}|\nabla \widetilde{v}|^{2} d x\right)^{2}\right) \\
\leq & t^{4} \int_{\mathbb{R}^{3}}\left(a_{\infty} f(\widetilde{v}) \widetilde{v}+\widetilde{v}^{6}\right) d x,
\end{aligned}
$$

a contradiction. Thus $t \leq 1$. Combining this with 2.22, 2.17), 2.16 and using Fatou's 
lemma, we obtain

$$
\begin{aligned}
c+o(1) & \geq \Psi\left(\widetilde{v}_{n}\right)-\frac{1}{4}\left\langle\Phi^{\prime}\left(\widetilde{v}_{n}\right), \widetilde{v}_{n}\right\rangle \\
& =\frac{1}{4}\left\|\widetilde{v}_{n}\right\|_{V_{\infty}}^{2}+\int_{\mathbb{R}^{3}} a_{\infty}\left(\frac{1}{4} f\left(\widetilde{v}_{n}\right) \widetilde{v}_{n}-F\left(\widetilde{v}_{n}\right)\right) d x+\frac{1}{12} \int_{\mathbb{R}^{3}}\left|\widetilde{v}_{n}\right|^{6} d x \\
& \geq \frac{1}{4}\|\widetilde{v}\|_{V_{\infty}}^{2}+\int_{\mathbb{R}^{3}} a_{\infty}\left(\frac{1}{4} f(\widetilde{v}) \widetilde{v}-F(\widetilde{v})\right) d x+\frac{1}{12} \int_{\mathbb{R}^{3}}|\widetilde{v}|^{6} d x+o(1) \\
& \geq \frac{1}{4}\|t \widetilde{v}\|_{V_{\infty}}^{2}+\int_{\mathbb{R}^{3}} a_{\infty}\left(\frac{1}{4} f(t \widetilde{v}) t \widetilde{v}-F(t \widetilde{v})\right) d x+\frac{1}{12} \int_{\mathbb{R}^{3}}|t \widetilde{v}|^{6} d x+o(1) \\
& =I^{\infty}(t \widetilde{v})-\frac{1}{4}\left\langle I^{\infty \prime}(t \widetilde{v}), t \widetilde{v}\right\rangle+o(1)=I^{\infty}(t \widetilde{v})+o(1) \geq m_{\infty}+o(1),
\end{aligned}
$$

a contradiction with the fact $c \in\left(0, m_{\infty}\right)$.

Now we consider the "vanishing" case. In this case, $v_{n} \rightarrow 0$ in $L^{s}\left(\mathbb{R}^{3}\right)(2<s<6)$, and then, by (2.5), we see that

$$
\int_{\mathbb{R}^{3}} a(x) F\left(v_{n}\right) d x \rightarrow 0 \text { and } \int_{\mathbb{R}^{3}} a(x) f\left(v_{n}\right) v_{n} d x \rightarrow 0
$$

as $n \rightarrow \infty$. Combining this and 2.20, 2.17), 2.16, we obtain

$$
\begin{aligned}
c+o(1) & \geq \Psi\left(v_{n}\right)=\frac{1}{2}\left\|v_{n}\right\|_{V_{\infty}}^{2}+\frac{b A}{4} \int_{\mathbb{R}^{3}}\left|v_{n}\right|^{2} d x-\frac{1}{6} \int_{\mathbb{R}^{3}}\left|v_{n}\right|^{6} d x, \\
o(1) & =\left\langle\Phi^{\prime}\left(v_{n}\right), v_{n}\right\rangle=\left\|v_{n}\right\|_{V_{\infty}}^{2}+b A \int_{\mathbb{R}^{3}}\left|\nabla v_{n}\right|^{2} d x-\int_{\mathbb{R}^{3}}\left|v_{n}\right|^{6} d x .
\end{aligned}
$$

Set $l=\lim _{n \rightarrow \infty} \int_{\mathbb{R}^{3}}\left|\nabla v_{n}\right|^{2} d x$. If $l>0$, then by 2.26 , we have

$$
\begin{aligned}
\int_{\mathbb{R}^{3}} a\left|\nabla v_{n}\right|^{2} d x+b\left(\int_{\mathbb{R}^{3}}\left|\nabla v_{n}\right|^{2} d x\right)^{2} & \leq \int_{\mathbb{R}^{3}}\left|v_{n}\right|^{6} d x+o(1) \\
& \leq S^{-3}\left(\int_{\mathbb{R}^{3}}\left|\nabla v_{n}\right|^{2} d x\right)^{3}+o(1)
\end{aligned}
$$

which implies that $l \geq\left(b S^{3}+\sqrt{\left(b S^{3}\right)^{2}+4 a S^{3}}\right) / 2$. Combining this and 2.25), (2.26), we deduce that

$$
\begin{aligned}
c+o(1) & \geq \frac{1}{3}\left\|v_{n}\right\|_{V_{\infty}}^{2}+\frac{b}{12}\left(\int_{\mathbb{R}^{3}}\left|\nabla v_{n}\right|^{2} d x\right)^{2} \\
& \geq \frac{a}{3} \int_{\mathbb{R}^{3}}\left|\nabla v_{n}\right|^{2} d x+\frac{b}{12}\left(\int_{\mathbb{R}^{3}}\left|\nabla v_{n}\right|^{2} d x\right)^{2} \\
& \geq \frac{a}{3}\left\{\frac{b S^{3}+\sqrt{\left(b S^{3}\right)^{2}+4 a S^{3}}}{2}\right\}+\frac{b}{12}\left\{\frac{b S^{3}+\sqrt{\left(b S^{3}\right)^{2}+4 a S^{3}}}{2}\right\}^{2}+o(1) \\
& =c^{*}+o(1),
\end{aligned}
$$

which contradicts $c<m_{\infty}<c^{*}$ (by Lemma 2.1). Thus $l=0$, which, jointly with 2.26), shows that $v_{n} \rightarrow 0$ in $E$. Therefore $u_{n} \rightarrow u$ in $E$. The proof is complete. 


\section{Proof of Theorem 1.1}

Proof of Theorem 1.1. The proof will be divided into three steps.

Step 1: I possesses a mountain pass geometry. For

$$
\varepsilon \in\left(0, \frac{1}{4}\left(\frac{1}{a}+\frac{1}{a S}\left|\int_{|x| \leq R_{0}} 1 d x\right|^{2 / 3}+\frac{2}{V_{\infty}}\right)^{-1}\right),
$$

by (2.4) and 2.1), we have

$$
\begin{aligned}
I(u) & \geq \frac{1}{2}\|u\|^{2}-\int_{\mathbb{R}^{3}}\left(\varepsilon|u|^{2}+C_{\varepsilon}|u|^{6}\right) d x-\frac{1}{6} \int_{\mathbb{R}^{3}}|u|^{6} d x \\
& \geq \frac{1}{4}\|u\|^{2}-\left(C_{\varepsilon}+\frac{1}{6}\right)(a M)^{-3}\|u\|^{6} .
\end{aligned}
$$

Thus there exist $\alpha, \rho>0$ such that $\left.I\right|_{\|u\|=\rho} \geq \alpha$. Choose $v \in E \backslash\{0\}$ with $v \geq 0$. By $\left(\mathrm{a}_{1}\right)$, we achieve that

$$
I(t v) \leq \frac{t^{2}}{2}\|v\|^{2}+\frac{b t^{4}}{4}\left(\int_{\mathbb{R}^{3}}|\nabla v|^{2} d x\right)^{2}-\frac{t^{6}}{6}\|v\|_{6}^{6} \rightarrow-\infty
$$

as $t \rightarrow+\infty$. So there exists $t_{0}>0$ large enough such that $I\left(t_{0} v\right)<0$ and $\left\|t_{0} v\right\| \geq \rho$. By virtue of the mountain pass theorem (see [2]), there exists a sequence $\left(u_{n}\right) \subset E$ such that $I\left(u_{n}\right) \rightarrow c>0$ and $I^{\prime}\left(u_{n}\right) \rightarrow 0$ as $n \rightarrow \infty$, where

$$
c=\inf _{\gamma \in \Gamma} \max _{t \in[0,1]} I(\gamma(t))
$$

with $\Gamma=\{\gamma \in C([0,1], E): \gamma(0)=0, I(\gamma(1))<0\}$.

Step 2: $c<m_{\infty}$. Let $R>0$ and $\beta=(1,0,0)$. It follows from the definition of $c$ that $c \leq \sup _{t \geq 0} I\left(t u_{\infty}(x-R \beta)\right)$. We claim that for $R>0$ large enough,

$$
\sup _{t \geq 0} I\left(t u_{\infty}(x-R \beta)\right)<m_{\infty}
$$

It follows from 2.1 and $\left(\mathrm{a}_{1}\right)$ that

$$
\begin{aligned}
I\left(t u_{\infty}(x-R \beta)\right) \leq & \frac{t^{2}}{2} \int_{\mathbb{R}^{3}}\left(a\left|\nabla u_{\infty}\right|^{2}+V_{M}\left|u_{\infty}\right|^{2}\right) d x \\
& +\frac{b t^{4}}{4}\left(\int_{\mathbb{R}^{3}}\left|\nabla u_{\infty}\right|^{2} d x\right)^{2}-\frac{t^{6}}{6} \int_{\mathbb{R}^{3}}\left|u_{\infty}\right|^{6} d x
\end{aligned}
$$

Thus there exist $t_{3}>0$ small and $t_{4}>0$ large such that

$$
\sup _{t \in\left[0, t_{3}\right] \cup\left[t_{4},+\infty\right]} I\left(t u_{\infty}(x-R \beta)\right)<m_{\infty}
$$


Observe that

$$
I\left(t u_{\infty}\right)=I^{\infty}\left(t u_{\infty}\right)+\frac{t^{2}}{2} \int_{\mathbb{R}^{3}}\left(V(x)-V_{\infty}\right)\left|u_{\infty}\right|^{2} d x-\int_{\mathbb{R}^{3}}\left(a(x)-a_{\infty}\right) F\left(t u_{\infty}\right) d x .
$$

Next we estimate the terms on the right-hand side. Choosing $\delta \in\left(0,1-b /\left(2 \sqrt{V_{\infty}}\right)\right)$, it follows from (2.3) and assumption $\left(\mathrm{V}_{2}\right)$ that

$$
\begin{aligned}
& \int_{|x| \geq R_{1}}\left(V(x)-V_{\infty}\right)\left|u_{\infty}(x-R \beta)\right|^{2} d x \\
\leq & \int_{|x| \geq R_{1}} C_{1} C_{\delta}^{2} e^{-b|x+R \beta|}\left|u_{\infty}(x)\right|^{2} d x \leq \int_{\mathbb{R}^{3}} C_{1} C_{\delta}^{2} e^{-b|x+R \beta|} e^{-2(1-\delta) \sqrt{V_{\infty}}|x|} d x \\
\leq & C_{1} C_{\delta}^{2} e^{-b R} \int_{\mathbb{R}^{3}} e^{\left(b-2(1-\delta) \sqrt{V_{\infty}}\right)|x|} d x \leq C_{4} e^{-b R} .
\end{aligned}
$$

Furthermore, by (2.1) and 2.3$)$, we have

$$
\begin{aligned}
& \int_{|x| \leq R_{1}}\left(V(x)-V_{\infty}\right)\left|u_{\infty}(x-R \beta)\right|^{2} d x \\
\leq & 2 M C_{\delta}^{2} \int_{|x| \leq R_{1}} e^{-2(1-\delta) \sqrt{V_{\infty}}|x-R \beta|} d x \\
\leq & 2 M C_{\delta}^{2} e^{-2(1-\delta) \sqrt{V_{\infty}} R} \int_{|x| \leq R_{1}} e^{2(1-\delta) \sqrt{V_{\infty}}|x|} d x \leq C_{5} e^{-2(1-\delta) \sqrt{V_{\infty}} R} .
\end{aligned}
$$

Choose $\widetilde{R}>0$ such that $\int_{|x| \leq \widetilde{R}}\left|u_{\infty}\right|^{p} d x>0$. Set $R>R_{2}+\widetilde{R}$. By $\left(\mathrm{a}_{2}\right)$ and $\left(\mathrm{f}_{2}\right)$, we obtain

$$
\begin{aligned}
& \int_{|x| \geq R_{2}}\left(a(x)-a_{\infty}\right) F\left(t u_{\infty}(x-R \beta)\right) d x \\
\geq & \frac{C_{2} C_{3} t_{3}^{p}}{p} \int_{|x| \geq R_{2}} e^{-d|x|}\left|u_{\infty}(x-R \beta)\right|^{p} d x \geq C \int_{|x-R \beta| \leq \widetilde{R}} e^{-d|x|}\left|u_{\infty}(x-R \beta)\right|^{p} d x \\
= & C \int_{|x| \leq \widetilde{R}} e^{-d|x+R \beta|}\left|u_{\infty}(x)\right|^{p} d x \geq C_{6} e^{-d R}
\end{aligned}
$$

for $t \in\left[t_{3}, t_{4}\right]$. Noting $|F(u)| \leq C\left(|u|^{2}+|u|^{6}\right)$ for all $u \in \mathbb{R}$, we derive that for $t \in\left[t_{3}, t_{4}\right]$,

$$
\begin{aligned}
& \left|\int_{|x| \leq R_{2}}\left(a(x)-a_{\infty}\right) F\left(t u_{\infty}(x-R \beta)\right) d x\right| \\
\leq & C C_{\delta}^{2} t_{4}^{2} \int_{|x| \leq R_{2}} e^{-2(1-\delta) \sqrt{V_{\infty}}|x-R \beta|} d x+C C_{\delta}^{6} t_{4}^{6} \int_{|x| \leq R_{2}} e^{-6(1-\delta) \sqrt{V_{\infty}}|x-R \beta|} d x \\
\leq & C C_{\delta}^{2} e^{-2(1-\delta) \sqrt{V_{\infty}} R} \int_{|x| \leq R_{2}} e^{2(1-\delta) \sqrt{V_{\infty}}|x|} d x+C C_{\delta}^{6} e^{-6(1-\delta) \sqrt{V_{\infty}} R} \int_{|x| \leq R_{2}} e^{6(1-\delta) \sqrt{V_{\infty}}|x|} d x \\
\leq & C_{7} e^{-2(1-\delta) \sqrt{V_{\infty}} R} .
\end{aligned}
$$


For $t>0$, set

$$
\begin{aligned}
g(t)= & I^{\infty}\left(t u_{\infty}\right) \\
= & \frac{t^{2}}{2} \int_{\mathbb{R}^{3}}\left(a\left|\nabla u_{\infty}\right|^{2}+V_{\infty} u_{\infty}^{2}\right) d x+\frac{b t^{4}}{4}\left(\int_{\mathbb{R}^{3}}\left|\nabla u_{\infty}\right|^{2} d x\right)^{2} \\
& -\int_{\mathbb{R}^{3}}\left(a_{\infty} F\left(t u_{\infty}\right)+\frac{t^{6}}{6} u_{\infty}^{6}\right) d x .
\end{aligned}
$$

Since

$$
\begin{aligned}
g^{\prime}(t)= & t\left[\int_{\mathbb{R}^{3}}\left(a\left|\nabla u_{\infty}\right|^{2}+V_{\infty} u_{\infty}^{2}\right) d x+t^{2} b\left(\int_{\mathbb{R}^{3}}\left|\nabla u_{\infty}\right|^{2} d x\right)^{2}\right] \\
& -t^{3}\left[\int_{\mathbb{R}^{3}} a_{\infty} \frac{f\left(t u_{\infty}\right)}{\left(t u_{\infty}\right)^{3}} u_{\infty}^{4} d x+t^{2} \int_{\mathbb{R}^{3}}\left|u_{\infty}\right|^{6} d x\right]
\end{aligned}
$$

and $f\left(t u_{\infty}\right) /\left(t u_{\infty}\right)^{3}$ is increasing for $t>0$, we deduce that $g(t)$ admits a unique critical point corresponding to its maximum. Noticing $g^{\prime}(1)=\left\langle I^{\infty}\left(u_{\infty}\right), u_{\infty}\right\rangle=0$, one has

$$
\sup _{t \geq 0} g(t)=g(1)=I^{\infty}\left(u_{\infty}\right)=m_{\infty} .
$$

Hence, combining (3.3)-(3.8), we obtain that

$$
\begin{aligned}
\sup _{t \in\left[t_{3}, t_{4}\right]} I\left(t u_{\infty}(x-R \beta)\right) \leq & \sup _{t \geq 0} I^{\infty}\left(t u_{\infty}(x-R \beta)\right)+\frac{t_{4}^{2}}{2}\left(C_{4} e^{-b R}+C_{5} e^{-2(1-\delta) \sqrt{V_{\infty}} R}\right) \\
& -C_{6} e^{-d R}-C_{7} e^{-2(1-\delta) \sqrt{V_{\infty}} R} \\
\leq & m_{\infty}+\widetilde{C}_{4} e^{-b R}+\widetilde{C}_{5} e^{-2(1-\delta) \sqrt{V_{\infty}} R}-C_{6} e^{-d R} .
\end{aligned}
$$

Since $0<d<b<2 \sqrt{V_{\infty}}$, we can find $R_{3}>R_{2}+\widetilde{R}$ sufficiently large such that

$$
\sup _{t \in\left[t_{3}, t_{4}\right]} I\left(t u_{\infty}\left(x-R_{3} \beta\right)\right)<m_{\infty}
$$

which, jointly with (3.2), shows that (3.1) holds. Therefore, $c \leq \sup _{t \geq 0} I\left(t u_{\infty}(x-R \beta)\right)<$ $m_{\infty}$. In view of Lemma 2.2, $u_{n} \rightarrow u$ in $E, I(u)=c$ and $I^{\prime}(u)=0$.

Step 3. Let

$$
m=\inf \{I(v): v \in M\}
$$

with $M=\left\{v \in E \backslash\{0\}: I^{\prime}(v)=0\right\}$. From Step 2, one has $u \in M$ and $m \leq I(u)<m_{\infty}$. For every $v \in M$ and $\varepsilon \in\left(0, \frac{1}{4}\left(\frac{1}{a}+\frac{1}{a S}\left|\int_{|x| \leq R_{0}} 1 d x\right|^{2 / 3}+\frac{2}{V_{\infty}}\right)^{-1}\right)$, we have

$$
\begin{aligned}
\|v\|^{2} & \leq \int_{\mathbb{R}^{3}}\left(a(x) f(v) v+v^{6}\right) d x \leq \varepsilon \int_{\mathbb{R}^{3}} v^{2} d x+\left(C_{\varepsilon}+1\right) \int_{\mathbb{R}^{3}}|v|^{6} d x \\
& \leq \frac{1}{4}\|v\|^{2}+\left(C_{\varepsilon}+1\right)(a S)^{-3}\|v\|^{6}
\end{aligned}
$$


by (2.4) and (2.2), which implies that $\|v\| \geq C_{0}$ for some $C_{0}>0$. Then $m>0$, i.e., $m \in\left(0, m_{\infty}\right)$. By the definition of $m$, there exist $\left(v_{n}\right) \in E \backslash\{0\}$ such that $I\left(v_{n}\right) \rightarrow m$ and $I^{\prime}\left(v_{n}\right)=0$. Applying Lemma 2.2, we deduce that $v_{n} \rightarrow v$ in $E, I(v)=m$ and $I^{\prime}(v)=0$. This completes the proof.

\section{Acknowledgments}

This work is partially supported by the National Natural Science Foundation of China (No. 11601049), Chongqing Natural Science Foundation and Frontier Research Planning Project (No. cstc2015jcyjA00014), and the Scientific and Technological Research Program of Chongqing Municipal Education Commission (No. KJ1500313).

\section{References}

[1] C. O. Alves and G. M. Figueiredo, Nonlinear perturbations of a periodic Kirchhoff equation in $\mathbb{R}^{N}$, Nonlinear Anal. 75 (2012), no. 5, 2750-2759.

[2] A. Ambrosetti and P. H. Rabinowitz, Dual variational methods in critical point theory and applications, J. Functional Analysis 14 (1973), 349-381.

[3] J. Chen, Multiple positive solutions to a class of Kirchhoff equation on $\mathbb{R}^{3}$ with indefinite nonlinearity, Nonlinear Anal. 96 (2014), 134-145.

[4] G. M. Figueiredo, Existence of a positive solution for a Kirchhoff problem type with critical growth via truncation argument, J. Math. Anal. Appl. 401 (2013), no. 2, 706-713.

[5] Y. He, G. Li and S. Peng, Concentrating bound states for Kirchhoff type problems in $\mathbb{R}^{3}$ involving critical Sobolev exponents, Adv. Nonlinear Stud. 14 (2014), no. 2, 483-510.

[6] Y. Huang, Z. Liu and Y. Wu, On finding solutions of a Kirchhoff type problem, Proc. Amer. Math. Soc. 144 (2016), no. 7, 3019-3033.

[7] G. Kirchhoff, Mechanik, Teubner, Leipzig, Germany, 1883.

[8] G. Li and H.-S. Zhou, The existence of a positive solution to asymptotically linear scalar field equations, Proc. Roy. Soc. Edinburgh Sect. A 130 (2000), no. 1, 81-105.

[9] L. Li and J.-J. Sun, Existence and multiplicity of solutions for the Kirchhoff equations with asymptotically linear nonlinearities, Nonlinear Anal. Real World Appl. 26 (2015), 391-399. 
[10] J.-L. Lions, On some questions in boundary value problems of mathematical physics, in: Contemporary Developments in Continuum Mechanics and Partial Differential Equations (Proc. Internat. Sympos., Inst. Mat., Univ. Fed. Rio de Janeiro, Rio de Janeiro, 1977), 284-346, North-Holland Math. Stud. 30, North-Holland, Amsterdam, 1978.

[11] P.-L. Lions, Solutions of Hartree-Fock equations for Coulomb systems, Comm. Math. Phys. 109 (1987), no. 1, 33-97.

[12] Z. Liu and S. Guo, On ground states for the Kirchhoff-type problem with a general critical nonlinearity, J. Math. Anal. Appl. 426 (2015), no. 1, 267-287.

[13] Z. Liu and C. Luo, Existence of positive ground state solutions for Kirchhoff type equation with general critical growth, Topol. Methods Nonlinear Anal. 49 (2017), no. $1,165-182$.

[14] D. Naimen, Positive solutions of Kirchhoff type elliptic equations involving a critical Sobolev exponent, NoDEA Nonlinear Differential Equations Appl. 21 (2014), no. 6, $885-914$.

[15] _ The critical problem of Kirchhoff type elliptic equations in dimension four, J. Differential Equations 257 (2014), no. 4, 1168-1193.

[16] S. I. Pohožaev, A certain class of quasilinear hyperbolic equations, Mat. Sb. (N.S.) 96(138) (1975), 152-166.

[17] J. Wang, L. Tian, J. Xu and F. Zhang, Multiplicity and concentration of positive solutions for a Kirchhoff type problem with critical growth, J. Differential Equations 253 (2012), no. 7, 2314-2351.

[18] M. Willem, Minimax Theorems, Progress in Nonlinear Differential Equations and Their Applications 24, Birkhäuser Boston, Boston, MA, 1996.

[19] Q. Xie, Singular perturbed Kirchhoff type problem with critical exponent, J. Math. Anal. Appl. 454 (2017), no. 1, 144-180.

[20] Q.-L. Xie, X.-P. Wu and C.-L. Tang, Existence and multiplicity of solutions for Kirchhoff type problem with critical exponent, Commun. Pure Appl. Anal. 12 (2013), no. 6, 2773-2786.

[21] L. Yang, Z. Liu and Z. Ouyang, Multiplicity results for the Kirchhoff type equations with critical growth, Appl. Math. Lett. 63 (2017), 118-123. 
[22] Y. Ye and C.-L. Tang, Multiple solutions for Kirchhoff-type equations in $\mathbb{R}^{N}$, J. Math. Phys. 54 (2013), no. 8, 081508, 16 pp.

[23] J. Zhang, On the Schrödinger equations with a nonlinearity in the critical growth, Topol. Methods Nonlinear Anal. 44 (2014), no. 2, 457-469.

[24] Real World Appl. 28 (2016), 153-170.

[25] J. Zhang and W. Zou, Multiplicity and concentration behavior of solutions to the critical Kirchhoff-type problem, Z. Angew. Math. Phys. 68 (2017), no. 3, Art. 57, 27 pp.

[26] X.-J. Zhong and C.-L. Tang, Multiple positive solutions to a Kirchhoff type problem involving a critical nonlinearity, Comput. Math. Appl. 72 (2016), no. 12, 2865-2877.

[27] X. P. Zhu and D. M. Cao, The concentration-compactness principle in nonlinear elliptic equations, Acta Math. Sci. (English Ed.) 9 (1989), no. 3, 307-328.

Yiwei Ye

School of Mathematical Sciences, Chongqing Normal University, Chongqing 401331, P. R. China

E-mail address: yeyiwei2011@126.com 